\section{Prokaryotic and eukaryotic cell-cycle proteins}

SIR-We report here what we believe to be the first demonstration of homology between prokaryotic and eukaryotic cellcycle proteins. It is the result of a comparison of the predicted amino-acid sequence of the Escherichia coli cell-cycle protein Fts $A^{\prime}$ determined by Robinson et al. ${ }^{2}$ with the National Biomedical Research Foundation protein sequence database (Version $8 ; 809,386$ residues) using the 'best local similarity' algorithm of Smith and Waterman $^{3}$ as implemented on an ICL DAP supercomputer by Lyall et al. ${ }^{4}$. A search of this type can give valuable insights into the possible functions and origins of predicted gene products as similarity of sequence suggests similarity of structure and function.

Alignments of amino-acid sequence are selected by the program from all those possible, including insertions and deletions where required in order to improve the quality of the alignment. Insertions and deletions are penalized to be worse than the worst mismatch scored. The analysis revealed a highly significant similarity, extending over a region of 60 amino acids, between the primary sequence of FtsA and the cell-cycle proteins CDC28 from the budding yeast Saccharomyces cerevisiae ${ }^{5}$ and $\mathrm{CDC} 2$ from the fission yeast Schizosaccharomyces pomben figure shows the extent of similarity observed between the three proteins.

We used the conserved elements in the region shown as a pattern to re-search the database for related segments and to assess their significance. The alignment of the conserved pattern with $\mathrm{CDC} 28$, for example, scored more than 60 standard deviations above the expected frequency, based on statistical analysis of the distribution of the scores of the best alignments. The homology reported between these proteins is therefore extremely significant; the next best alignment scored only 2 standard deviations above expectation, and is not significant. There is no significant homology to human $\mathrm{CDC} 2 \mathrm{Hs}^{7}$

What might be the reason for this striking similarity between $E$. coli and yeast cell-cycle proteins? The functions of these proteins (other than the kinase activities

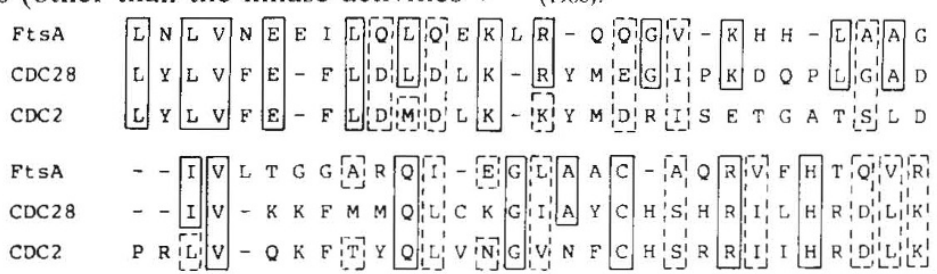

Comparison of amino-acid sequences from segments of the FtsA protein of $E$. coli $^{2}$ (residues $305-357$ ), the CDC28 protein of $S$. cerevisiae (residues 84-138) and the CDC2 protein of $S$. pombe $e^{6}$ (residues 80-136). The sequences are aligned for maximum homology ${ }^{4}$. Amino-acids which are identical in FtsA and one or both of CDC 28 and CDC2 are shown in solid-lined boxes, with conservative amino-acid changes in dashed-lined boxes. Related amino acids are grouped as follows: P,A,G,S,T - neutral, weakly hydrophobic; Q,N,E,D - hydrophilic acid amine; H,K, R — hydrophilic, basic; L,I,V,M - hydrophobic; F, Y,W - hydrophobic, aromatic.

\section{Fertilization events}

SIR-Dale ${ }^{1}$ misrepresents the state of knowledge on the mechanism of fertilization. The existence of a rapid electrical block to polyspermy is solidly supported by data from sea urchins, starfish, the marine worm Urechis and frogs, where it has been shown that a rapid positive-going shift in the egg membrane potential occurs in response to insemination, that positive membrane potentials during insemination inhibit sperm penetration, and that negative potentials promote polyspermy ${ }^{2.3}$. The recent experiments ${ }^{4-7}$ cited by Dale do not cast doubt on this hypothesis.

In fact, Shen and Steinhardt ${ }^{4}$ confirmed that positive potential inhibits sperm entry and negative potentials promote it: when periods of positive potential were interrupted by transient $(10-80 \mathrm{~ms})$ shifts to negative potentials, the more negative the potential, the more probable was fertilization. Although 2-3 dozen sperm were bound to an egg at the time the negative voltage windows were applied, only 43 out of 145 eggs were fertilized and most of these were monospermic. This does not contradict the existence of an electrical polyspermy block, because the more negative the voltage and the longer the duration of the voltage window, the more eggs were fertilized, as would be predicted.

Furthermore, the incidence of polyspermy during the negative voltage windows was not less than expected. Eighteen of the 43 eggs that were fertilized during the voltage windows were scored for polyspermy: one was dispermic and the rest monospermic. When the data are analysed by the Poisson distribution an expected frequency of 3 per cent is calculated for dispermic eggs close to the observed frequency of about 2 per cent.

Dale himself states of his data" "The present report does not directly address the question of a fast block". In the other experiments $^{6,7}$, he cites sperm concentrations were deliberately kept low to avoid the polyspermy that would otherwise occur in eggs voltage-clamped to $-20 \mathrm{mV}$. Thus there are no recent data that cast doubt on the occurrence of an electrical polyspermy block. To avoid confusion it should be pointed out that while some species possess electrical polyspermy blocks, others do not. Exceptions include hamster, the fish Oryzias, and salamanders (see ref. 2).

The other question raised by Dale was how sperm initiate egg activation. He suggested that if fusion and the first event in egg activation are simultaneous then it is more likely that the sperm injects materials into the egg through the cytoplasmic bridge formed at sperm-egg fusion than that there is the type of interaction in which sperm act as a ligand and the egg as a receptor. This is not logical. If fusion and the initiation of activation occur at the 